\title{
Níveis de concentrado e sais de cálcio de ácidos graxos para novilhos terminados em confinamento
}

\author{
Concentrate levels and calcium salts of fatty acids for feedlot finished steers
}

\author{
Regina Célia Cardoso Margarido ${ }^{\mathrm{I}}$ Paulo Roberto Leme ${ }^{\mathrm{II}}$ Saulo da Luz e Silva ${ }^{\mathrm{I}^{*}}$ \\ Angélica Simone Cravo Pereira ${ }^{\mathrm{II}}$
}

\section{RESUMO}

Objetivou-se avaliar o efeito do nível de concentrado e dos sais de cálcio de ácidos graxos (SCAG) sobre o desempenho e qualidade da carne de novilhos terminados em confinamento. Sessenta novilhos Nelore e cruza Nelore foram divididos em quatro grupos e confinados por um período de 85 dias com as seguintes dietas: (BC) 46,7\% de concentrado; (BC-SCAG) dieta BC acrescida de 3\% de SCAG; $(A C)$ dieta contendo 76,6\% de concentrado e (AC-SCAG) dieta AC com $3 \%$ de SCAG. O nível de concentrado não influenciou o ganho médio diário, a espessura de gordura subcutânea e a área de olho de lombo, mas as dietas AC aumentaram o peso $(P=0,0011)$ e o rendimento $(P<0,0001)$ de carcaça, além da força de cisalhamento $(P=0,0438)$. Animais alimentados com as dietas AC apresentaram maior peso $(P=0,0011) e$ rendimento $(P<0,0001)$ de carcaça em relação aos animais do tratamento BC. Os SCAG aumentaram o ganho de peso em dietas de $A C(P=0,0311)$, mas sem efeito nas dietas BC. Animais tratados com as dietas contendo SCAG apresentaram maior peso $(P=0,0133)$ e rendimento de carcaça $(P=0,0160)$, mas sem diferenças na espessura de gordura subcutânea, área de olho de lombo e força de cisalhamento. Dietas de alto concentrado e os SCAG melhoraram as características quantitativas (peso e rendimento) das carcaças de bovinos na fase de terminação e podem ser utilizadas pelos produtores como alternativa para melhorar a eficiência do sistema de produção.

Palavras-chave: bovinos de corte, características de carcaça, desempenho em confinamento, gordura vegetal protegida.

\section{ABSTRACT}

The objective of this research was to evaluate the effects of concentrate level and calcium salts of fatty acids
(SCAG) on performance and meat quality of feedlot finished steers. Sixty Nellore and Nellore crossbred steers were distributed in four groups and fed for 85 days with one of the following diets: $B C$ ) diet containing $46.7 \%$ of concentrate; (BC-SCAG) $L C$ diet plus $3 \%$ of SCAG; (AC) diet containing $76.6 \%$ of concentrate and (AC-SCAG) HC diet plus $3 \%$ of SCAG. Concentrate level did not affected average daily gain, subcutaneous fat thickness and ribeye area, but AC diets increased carcass weight $(P=0.0011)$, dressing percentage $(P<0.0001)$ and shear force $(P=0.0438)$. SCAG increased average daily gain in $A C(P=0.0311)$ but not in $B C$ diets. Animals fed with SCAG diets showed greater carcass weight $(P=0.0133)$ and dressing percentage $(P=0.0160)$ with no differences on fat thickness, ribeye area and shear force. High concentrate diets and SCAG improved quantitative carcass traits (weight and dressing percentage) of finishing cattle and can be used as alternative to improve the efficiency of the production system.

Key words: beef cattle, carcass traits, feedlot performance, protected vegetable fat.

\section{INTRODUÇÃO}

No Brasil, dietas para confinamento tradicionalmente foram balanceadas com alta proporção de volumosos, devido ao alto custo dos grãos e concentrados proteicos, quando comparados a dietas à base de forragens. Entretanto, esse cenário tem apresentado algumas mudanças, principalmente com a utilização de dietas com maiores teores de

ILDC-SEV Bioenergia S/A, Orlândia, SP, Brasil.

"Departamento de Zootecnia, Faculdade de Zootecnia e Engenharia de Alimentos (FZEA), Universidade de São Paulo (USP), 13.635-900, Pirassununga, SP, Brasil. E-mail: sauloluz@usp.br. *Autor para correspondência

II'Departamento de Nutrição e Produção Animal, Faculdade de Medicina Veterinária e Zootecnia (FMVZ), USP, Pirassununga, SP, Brasil. 
concentrado. Segundo MILLEN et al. (2009), cerca de $58 \%$ dos nutricionistas responsáveis por confinamentos no Brasil utilizam dietas com mais de $70 \%$ de concentrado, para bovinos em fase de terminação. A utilização desse tipo de dieta em confinamento torna-se viável economicamente com a utilização de subprodutos de baixo custo e quando os grãos apresentam preço vantajoso (LEME et al., 2003), pois proporcionam um maior ganho de peso, maior eficiência alimentar e redução nos custos com mão-deobra, tornando a atividade mais rentável (BULLE et al., 2002).

O fornecimento de dietas com elevado teor de concentrado para bovinos possibilita o abate de animais jovens com acabamento de gordura adequado, melhorando a qualidade da carne e aumentando o peso e o rendimento de carcaça (OWENS \& GARDNER, 1999), características utilizadas como referência para classificação e remuneração das carcaças na indústria frigorífica. No entanto, o aumento da densidade energética da dieta através da adição de carboidratos pode apresentar alguns problemas, pois animais zebuínos apresentam desempenhos inferiores aos estimados quando são alimentados com dietas em que o amido responde por mais de $45 \%$ da matéria seca, ou quando o teor de concentrado excede $75 \%$ da matéria seca (ALMEIDA et al., 2010). Com o objetivo de aumentar a densidade energética da dieta, melhorando a eficiência alimentar, além de garantir a ingestão de fibra necessária para o bom funcionamento do rúmen, gorduras e óleos têm sido utilizados em substituição a elevadas proporções de grãos (VALINOTE et al., 2005). No entanto, o fornecimento de altos níveis de gordura na dieta pode causar problemas de absorção de nutrientes, pois os ácidos graxos reduzem o $\mathrm{pH}$ do rúmen e, consequentemente, alteram a flora ruminal, prejudicando a digestibilidade dos alimentos (AFERRI et al., 2005).

Uma alternativa seria o fornecimento de gordura protegida da biohidrogenação ruminal, que não interfere no processo digestivo ruminal, sendo dissolvida e absorvida no intestino delgado. No entanto, há pouca informação disponível sobre os efeitos dos sais de cálcio de ácidos graxos (SCAG) na alimentação de bovinos de corte terminados em confinamento e seus efeitos sobre o desempenho, o rendimento e a qualidade da carcaça e da carne. Dessa forma, objetivou-se avaliar o efeito do nível de concentrado da dieta e da adição de SCAG sobre o desempenho e a qualidade da carne de bovinos confinados na fase de terminação.

\section{MATERIAL E MÉTODOS}

Foram utilizados 60 novilhos cruzados (Nelore x Nelore, Simental, Charolês, Pardo Suiço ou Red Angus), com peso médio inicial de 367 $33,5 \mathrm{~kg}$ e 36 meses de idade. Os animais foram distribuídos em quatro baias (15 animais por baia), de forma a manter a proporção de animais com grau de sangue semelhante em cada um dos piquetes (tratamentos). Os piquetes continham cocho e bebedouro a céu aberto e área de $438 \mathrm{~m}^{2}$ (17,5x25m) cada. Após um período de adaptação de 15 dias às instalações e às dietas, os animais foram confinados com uma das seguintes dietas: (BC) dieta com baixo concentrado; (BC-SCAG) dieta com baixo concentrado, acrescida de 3\% de SCAG; (AC) dieta com alto concentrado; e (AC-SCAG) dieta com alto concentrado com 3\% de SCAG (Tabela 1). A fonte de SCAG utilizada foi o produto comercial Lactoplus ${ }^{\circledR}$ (Dalquim Indústria Química Ltda, Itajaí, SC, Brasil). A alimentação foi fornecida uma vez ao dia e as sobras retiradas e pesadas diariamente para realização do ajuste no total de alimento a ser oferecido e para o cálculo do consumo de matéria seca e conversão alimentar do lote.

As pesagens foram realizadas após jejum completo de aproximadamente 18 horas e a cada intervalo de 28 dias. $\mathrm{O}$ abate dos animais foi realizado após 85 dias de confinamento em abatedouro comercial, quando a média da espessura de gordura subcutânea, medida por ultrassom, na região entre a $12^{\mathrm{a}}$ e a $13^{\mathrm{a}}$ costelas, atingiu cinco milímetros. As avaliações por ultrassonografia foram realizadas utilizando um equipamento marca Aloka, modelo SSD 500 Micrus (Aloka Co. Ltd.), com transdutor linear de 3,5mHz e 172 $\mathrm{mm}$ de comprimento, com guia acústica acoplada. As imagens obtidas foram armazenadas em um microcomputador portátil e posteriormente analisadas, utilizando o software Lince $^{\circledR}$. A determinação da gordura subcutânea foi realizada a $3 / 4$ da borda medial do músculo Longissimus.

Após o abate, foi obtido o peso da carcaça quente (PCQ) e depois elas foram resfriadas por $24 \mathrm{~h}$ a uma temperatura entre $0^{\circ} \mathrm{Ce} 2^{\circ} \mathrm{C}$. Em seguida, a meiacarcaça esquerda foi dividida entre a $12^{\mathrm{a}}$ e a $13^{\mathrm{a}}$ costelas, para determinação da área de olho de lombo (AOL) e espessura de gordura subcutânea (EGS) e para retirada de três amostras de $2,5 \mathrm{~cm}$ de espessura do músculo Longissimus, na região entre a $11^{\underline{a}}$ e a $12^{\mathrm{a}}$ costelas, para determinação da força de cisalhamento (FC). As amostras foram identificadas e embaladas a vácuo em filme flexível de baixa permeabilidade de oxigênio e maturadas por 0,7 ou 14 dias, a uma temperatura entre 0 e $2^{\circ} \mathrm{C}$. Após esse período, foram armazenadas em 
Tabela 1 - Composição percentual das dietas utilizadas (\% da matéria seca) com baixo concentrado (BC), BC com sais de cálcio de ácidos graxos (BC-SCAG), alto concentrado (AC) e AC com sais de cálcio de ácidos graxos (AC-SCAG), expressos em matéria seca.

\begin{tabular}{lllcc}
\hline & \multicolumn{3}{c}{ Tratamentos } \\
Ingredientes & BC & AC & BC-SCAG & AC-SCAG \\
\hline Bagaço de cana-de-açúcar hidrolisado & 48,640 & 13,360 & 48,640 & 13,360 \\
Bagaço de cana-de-açúcar in natura & 4,480 & 10,000 & 4,480 & 10,000 \\
Farelo de Soja & 7,890 & 6,000 & 7,890 & 6,000 \\
Levedura Úmida & 11,214 & 10,000 & 11,214 & 10,000 \\
Milho Grão & 21,729 & 35,213 & 18,729 & 32,213 \\
Casca de Soja & - & 20,000 & 20,000 \\
Melaço & 2,940 & 2,500 & 2,500 \\
Lactoplus & - & - & 3,000 \\
Uréia & 0,780 & 0,600 & 3,000 & 0,600 \\
Sal Mineral & 2,300 & 2,300 & 0,780 & 2,300 \\
Rumensin & & 0,027 & 2,300 & 0,027 \\
Nutrientes, $\%$ & 0,027 & & 0,027 & 13,9 \\
Proteína bruta & & 13,9 & 12,1 & 76,3 \\
Nutrientes digestíveis totais ${ }^{3}$ & 12,1 & 71,4 & 67,3 & 0,61 \\
Ca, \% & 64,5 & 0,61 & 0,52 & 0,34 \\
P & 0,53 & 0,35 & 0,30 & \\
\hline
\end{tabular}

${ }^{1}$ Sais de cálcio de ácidos graxos (Dalquin Ind. Química Ltda); ${ }^{2}$ Monensina sódica a 10\% (Elanco Animal Health); ${ }^{3}$ estimado pela fórmula de WEISS et

freezer doméstico a $-18^{\circ} \mathrm{C}$ para posterior análise. A FC foi determinada através do equipamento Warner Bratzler Shear Force, conforme a metodologia recomendada pela AMSA (1995).

O delineamento experimental utilizado foi o inteiramente casualizado em arranjo fatorial $2 \times 2$ (nível de concentrado x SCAG). As pressuposições do modelo fixo foram verificadas antes da realização da análise de variância. Em virtude da diferença observada no peso inicial dos animais entre os lotes, o peso inicial foi utilizado como covariável no modelo para avaliação do peso final (PF), ganho médio diário (GMD), peso (PCQ) e rendimento (RCQ) de carcaça quente, AOLe EGS, conforme o modelo:

$$
y_{i j k}=\mu+C_{i}+G_{j}+C G_{i j}-\beta_{0}\left(P_{i j k}-P\right)+e_{i j k}, e m
$$

que $\mathrm{Y}_{\mathrm{ijk}}$ é a característica de desempenho do animal submetido ao i-ésimo nível de concentrado (1=alto; 2=baixo), e ao j-ésimo nível de SCAG (1=sem; $2=\mathrm{com}$ ) na k-ésima repetição; $\mu$ é a média geral; $\mathrm{C}_{\mathrm{i}}$ efeito do nível de concentrado $\mathrm{i} ; \mathrm{G}_{\mathrm{j}}$ efeito do SCAG j; $\mathrm{CG}_{\mathrm{ij}} \mathrm{o}$ efeito da interação entre o nível de concentrado e SCAG; $\beta_{0}$ é o coeficiente de regressão linear, incluído no modelo como covariável; $\mathrm{P}_{\mathrm{i} j \mathrm{k}}$ é o peso do indivíduo na k-ésima repetição; é a média aritmética dos pesos iniciais de todos os animais do experimento e $\mathrm{e}_{\mathrm{ijk}}$ o erro residual.

Quando não foi observado efeito significativo da covariável $(\mathrm{P}>0,05)$, ela foi retirada do modelo e os resultados foram apresentados sem ajuste.
A FC foi analisada como medida repetida no tempo (CROWDER \& HAND, 1990), sendo incluído no modelo o efeito do tempo de maturação, além dos fatores principais anteriormente mencionados, conforme o seguinte modelo:

$\mathrm{y}_{\mathrm{i} \mathrm{kl}}=\mu+\mathrm{C}_{\mathrm{i}}+\mathrm{G}_{\mathrm{j}}+\mathrm{CG}_{\mathrm{ij}}+\mathrm{T}_{\mathrm{k}} \mathrm{e}_{\mathrm{i} \mathrm{jk}}$, em que $\mathrm{Y}_{\mathrm{ijkl}}$ é a força de cisalhamento da carne do animal, submetido ao i-ésimo nível de concentrado (1=alto; $2=$ baixo), e ao j-ésimo nível de SCAG (1=sem; 2=com); no k-ésimo tempo de maturação ( $1=0$ dias; $2=7$ dias; $3=14$ dias) da l-ésima repetição; $\mu$ é a média geral; $\mathrm{C}_{\mathrm{i}}$ efeito do nível de concentrado $\mathrm{i} ; \mathrm{F}_{\mathrm{j}}$ efeito do $\mathrm{SCAG} \mathrm{j} ; \mathrm{T}_{\mathrm{k}}$ efeito do tempo de maturação; $\mathrm{CG}_{\mathrm{ijk}}$ o efeito da interação entre o nível de concentrado; e SCAG e e $e_{i \mathrm{jkl}}$ o erro residual.

Quando houve efeito significativo da interação, foi realizado o desdobramento e os resultados eito do tempo de maturação; $\mathrm{CG}_{\mathrm{ijk}}$ o efeito da interação entre o nível de concentrado; e SCAG e e $e_{i j k l}$ o erro residual, comparados dentro de cada fator através do teste T-Student. $\mathrm{O}$ efeito do grupo genético não foi considerado no modelo. As análises foram realizadas utilizando-se o procedimento MIXED, do software $\mathrm{SAS}^{\circledR}$ versão 8.2. (SAS Institute Inc., Cary, NC).

\section{RESULTADOS E DISCUSSÃO}

Foi observado efeito do peso inicial (covariável) sobre o PF, PCQ e RCQ $(\mathrm{P}<0,0001)$ e, 
portanto, os resultados para essas características foram ajustados para o peso inicial. Houve uma interação entre nível de concentrado, SCAG para o $\mathrm{PF}(\mathrm{P}=0,0135)$, entretanto, após o desdobramento da interação não foi verificada diferença entre os fatores e, portanto, são apresentados apenas os resultados para os fatores principais (Tabela 2). Não foi observado efeito do nível de concentrado ou dos SCAG sobre o PF.

A ingestão de matéria seca média do lote dos animais do tratamento BC foi de $11,7 \mathrm{~kg} \mathrm{dia}^{-1}$ e de $10,5 \mathrm{~kg} \mathrm{dia}^{-1}$ no tratamento AC. Nos animais alimentados com dietas contendo SCAG, o consumo médio foi de $10,9 \mathrm{~kg} \mathrm{dia}^{-1} \mathrm{e}$, naqueles alimentados com a dieta sem SCAG, o consumo médio foi de $11,3 \mathrm{~kg} \mathrm{dia}^{-1}$. A conversão alimentar ( $\mathrm{kg}$ alimento ingerido $\mathrm{kg}^{-1}$ ganho médio diário), calculada com base no consumo médio do lote, foi de $8,3 \mathrm{~kg}$ para os animais do tratamento AC e de $9,3 \mathrm{~kg}$ para os animais do tratamento BC. Nas dietas sem SCAG, a conversão alimentar foi de 9,0kg e de $8,6 \mathrm{~kg}$ naquelas com SCAG. Apesar de não ser possível fazer inferência estatística sobre os dados de ingestão de matéria seca, uma vez que os dados representam as médias dos lotes, é possível perceber uma redução na ingestão de alimento e na conversão alimentar dos animais alimentados com as dietas com alto concentrado.

Também foi observada uma interação entre nível de concentrado e SCAG para o GMD, (P=0,0124). Não houve diferença no GMD dos animais do tratamento BC $\left(1,33 \mathrm{~kg} \mathrm{dia}^{-1}\right)$ em relação aos animais do tratamento $\mathrm{AC}\left(1,24 \mathrm{~kg} \mathrm{dia}^{-1}\right)$. No entanto, animais alimentados com a dieta AC-SCAG apresentaram maior
$\mathrm{GMD}(\mathrm{P}=0,0101)$ em relação aos alimentados com a dieta BC-SCAG (1,44 e 1,20kg dia ${ }^{-1}$, respectivamente; Figura 1). Segundo OWENS \& GARDNER (1999), o aumento do nível de concentrado (em dietas com mais de $70 \%$ de concentrado) esteve associado a uma melhora na eficiência alimentar e ao aumento do GMD, até certo nível, determinado pelo limite biológico do animal. No entanto, é possível encontrar resultados contrastantes na literatura em relação ao efeito do nível de concentrado sobre o desempenho, principalmente em animais zebuínos. De acordo com PUTRINO et al. (2002), o aumento do nível de concentrado (20, 40, 60 e 80\%) esteve associado a um aumento linear do GMD. Por outro lado, LEME et al. (2003) não verificaram diferença no GMD de animais alimentados com dietas contendo altos níveis de concentrado $(73,79$ ou $85 \%)$.

Em relação ao efeito dos SCAG, foi observado um maior GMD $(\mathrm{P}=0,0311)$ para os animais que receberam a dieta AC-SCAG (1,44 kg/dia) em relação aos $\mathrm{AC}\left(1,20 \mathrm{~kg} \mathrm{dia}^{-1}\right)$, mas sem diferença entre os animais alimentados com as dietas BC e BC-SCAG (1,33 e $1,20 \mathrm{~kg} \mathrm{dia}^{-1}$, respectivamente). O maior GMD verificado nas dietas AC-SCAG pode ter ocorrido em função do maior aporte de energia nessa dieta $(76,3 \%$ NDT) em relação a AC $(71,45 \%)$, uma vez que o consumo médio dos grupos foi semelhante $\left(10,4\right.$ e 10,6 $\mathrm{kg} \mathrm{dia}^{-1}$, respectivamente; dados não mostrados).

A adição de gordura na dieta de bovinos de corte na fase de terminação tem mostrado diferentes respostas em relação ao desempenho e às características de carcaça. AFERRI et al. (2005) e SILVA et al. (2007) não observaram o efeito da inclusão de

Tabela 2 - Médias obtidas pelo método dos quadrados mínimos e erro padrão (EP) das características de desempenho e carcaça avaliadas.

\begin{tabular}{|c|c|c|c|c|c|c|}
\hline \multirow[t]{3}{*}{ Característica } & \multicolumn{6}{|c|}{ - } \\
\hline & \multicolumn{2}{|c|}{-----Nível de concentrado----- } & & \multicolumn{2}{|c|}{---------SCAG--------- } & \\
\hline & $\mathrm{BC}$ & $\mathrm{AC}$ & EP & Sem & Com & $\mathrm{EP}$ \\
\hline Peso final, $\mathrm{kg}$ & 475,9 & 480,3 & 5,63 & 477,1 & 479,0 & 6,98 \\
\hline Ingestão de matéria $\mathrm{seca}^{2}, \mathrm{~kg}$ & 11,7 & 10,5 & - & 11,3 & 10,9 & - \\
\hline Conversão alimentar ${ }^{3}, \mathrm{~kg}$ & 9,3 & 8,3 & - & 9,0 & 8,6 & - \\
\hline Rendimento de carcaça, \% & $55,4^{\mathrm{b}}$ & $58,4^{\mathrm{a}}$ & 0,36 & $56,4^{\mathrm{b}}$ & $57,5^{\mathrm{a}}$ & 0,28 \\
\hline Área de olho de lombo, $\mathrm{cm}^{2}$ & $64,4^{\mathrm{b}}$ & $76,7^{\mathrm{a}}$ & 1,72 & $66,7^{\mathrm{b}}$ & $74,4^{\mathrm{a}}$ & 1,33 \\
\hline Espessura de gordura, $\mathrm{mm}$ & 5,2 & 4,9 & 0,42 & 5,2 & 4,9 & 0,42 \\
\hline Força de cisalhamento - 0 dias, $\mathrm{kg}$ & $3,5^{\mathrm{b}}$ & $3,9^{\mathrm{a}}$ & 0,15 & 3,6 & 3,8 & 0,15 \\
\hline Força de cisalhamento - 7 dias, $\mathrm{kg}$ & 3,0 & 3,3 & 0,13 & 3,3 & 3,1 & 0,13 \\
\hline Força de cisalhamento - 14 dias, $\mathrm{kg}$ & 2,5 & 2,4 & 0,08 & 2,5 & 2,4 & 0,08 \\
\hline
\end{tabular}

${ }^{1} \mathrm{BC}$ - dieta com baixo concentrado; $\mathrm{AC}$ - dieta de alto concentrado; SCAG - sais de cálcio de ácidos graxos.

a,b Letras diferentes dentro de níveis de concentrado ou dos sais de cálcio de ácidos graxos (SCAG) diferem entre si pelo teste T- Student ao nível de probabilidade de $5 \%$. 


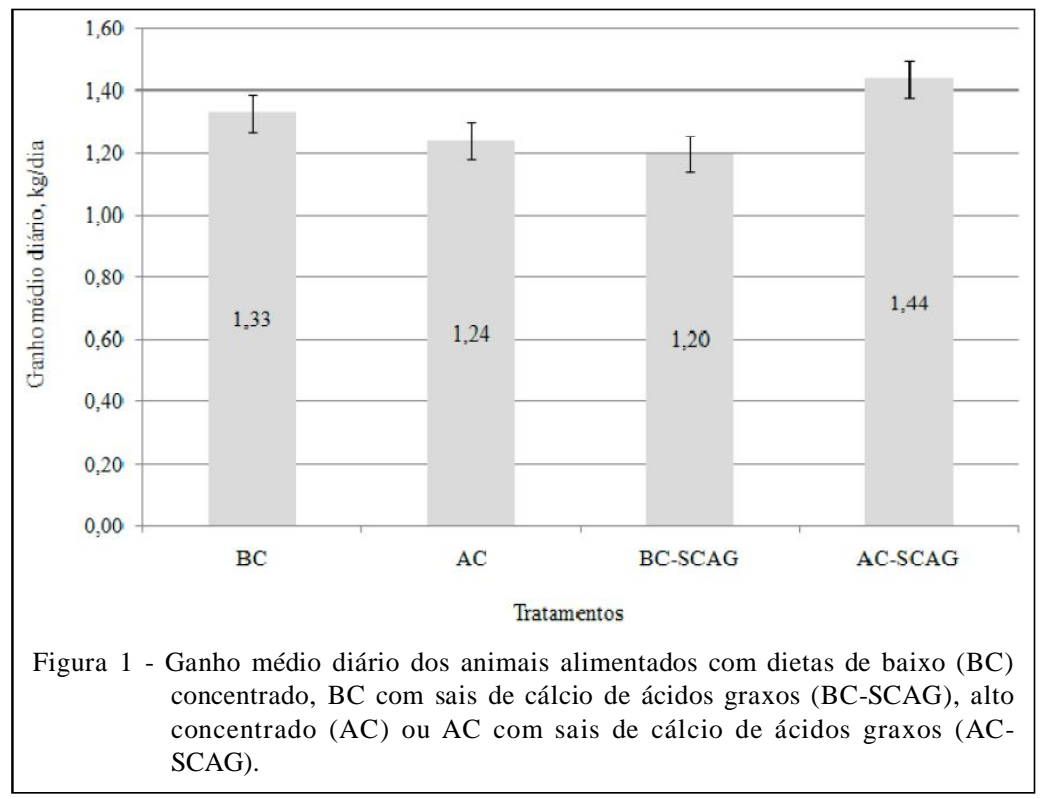

SCAG $\left(5 \%\right.$ de Lac $100^{\circledast}$ e $4 \%$ de Lactoplus ${ }^{\circledR}$, respectivamente) sobre o desempenho de bovinos confinados. Por outro lado, NGIDI et al. (1990) observaram uma redução linear na ingestão de matéria seca e no GMD, com o aumento do nível SCAG. Segundo OWENS \& GARDNER (1999), altos níveis de gordura na dieta tem sido associados à redução do GMD e melhora na eficiência alimentar.

Foi observada uma interação entre os níveis de concentrado e os SCAG para o PCQ ( $\mathrm{P}=0,0273)$.
Animais tratados com a dieta $\mathrm{AC}$ apresentaram maior $\mathrm{PCQ}(\mathrm{P}=0,0011$; Figura 2) em relação aos da dieta BC, assim como os do tratamento AC-SCAG apresentaram maior PCQ em relação aos $\mathrm{BC}-\mathrm{SCAG}(\mathrm{P}=0,0011)$.

Animais alimentados com dietas AC apresentaram um $\mathrm{RCQ} 3 \%$ maior $(\mathrm{P}<0,0001)$ em relação àqueles alimentados com dietas $\mathrm{BC}(58,4$ e $55,4 \%$, respectivamente), bem como uma maior $\mathrm{AOL}(76,7 \mathrm{e}$ $64,4 \mathrm{~cm}^{2}$, respectivamente; $\mathrm{P}=0,0002$; Tabela 2). AEGS não foi influenciada pelo nível de concentrado,

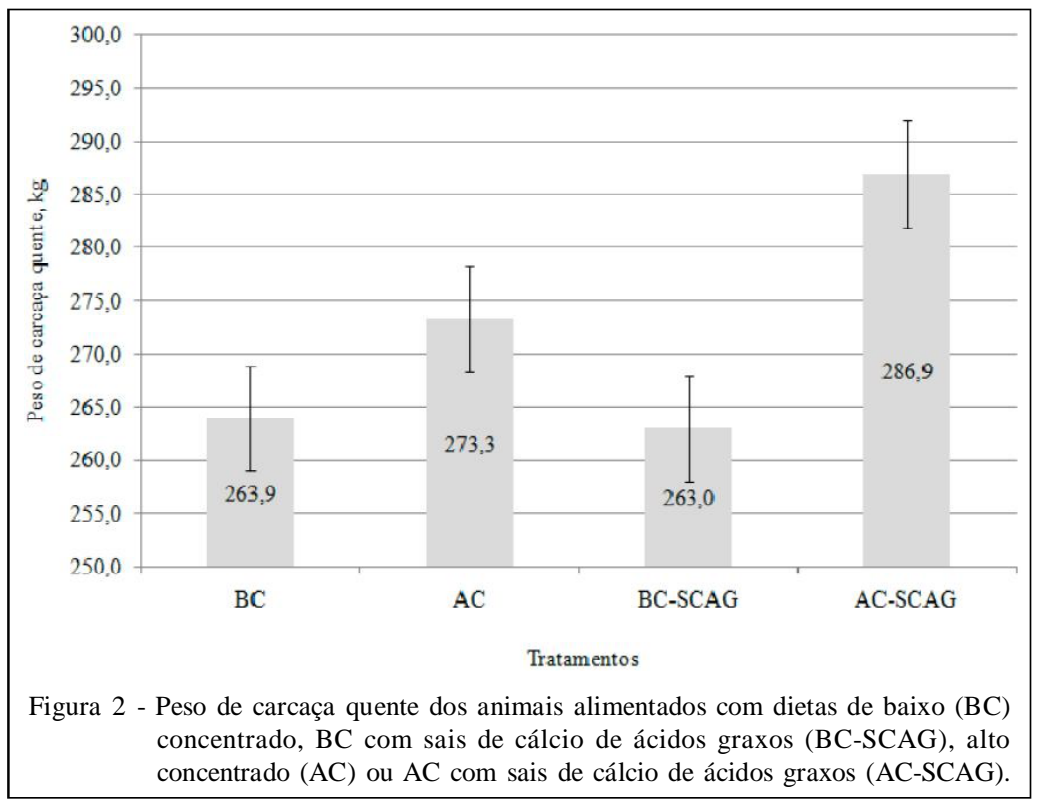

Ciência Rural, v.41, n.2, fev, 2011. 
conforme esperado, uma vez que os animais foram abatidos quando atingiram um grau de acabamento prédeterminado $(5 \mathrm{~mm})$. O maior RCQ observado neste trabalho para animais alimentados com dietas AC está de acordo com o observado por OWENS \& GARDNER (1999), os quais relataram que a maior porcentagem de concentrado esteve associada ao maior PCQ e RCQ. Resultados semelhantes foram observados por LEME et al. (2003), em novilhos Nelore, em que o aumento do nível de concentrado de $73 \%$ para $85 \%$ aumentou o RCQem 1,6\%.

Os SCAG não influenciaram o PCQ nas dietas de baixo concentrado, entretanto, ele foi maior nos animais do tratamento AC-SCAG $(\mathrm{P}=0,0133) \mathrm{em}$ relação aos animais do tratamento $\mathrm{AC}(286,9$ e $273,3 \mathrm{~kg}$,respectivamente). Esses dados são coerentes com relatados por OWENS \& GARDNER (1999), que verificaram um aumento do rendimento de carcaça com a adição de gordura na dieta, obtendo um maior RCQ estimado em dietas contendo $2,4 \%$ de gordura adicionada, semelhante ao nível utilizado neste trabalho. De acordo com os autores, a adição de $2 \%$ de gordura ou $4 \%$ na dieta total esteve associada com maior área de olho de lombo, pequenos incrementos na marmorização, menor espessura de gordura subcutânea e pior qualidade da carcaça. Por outro lado, os resultados observados neste trabalho diferem dos relatados por AFERRI et al. (2005), que não observaram efeito da adição de 5\% de SCAG sobre as características de carcaça de novilhos cruzados.

Além disso, NGIDI et al. (1990) descreveram um efeito negativo sobre o PCQ com o aumento do nível de SCAG na dieta, possivelmente devido à redução do consumo apresentado pelos animais alimentados com níveis mais elevados de SCAG, enquanto outras características de carcaça, como $\mathrm{AOL}$ eEGS, não foram influenciadas. Da mesma forma, ZINN et al. (2000) também não encontraram diferença na AOL e EGS de novilhos holandeses, alimentados com níveis de até $6 \%$ de SCAG na dieta.

Animais alimentados com dietas BC apresentaram menor $\mathrm{FC}(\mathrm{P}=0,0438)$ nas amostras sem maturação (tempo zero), comparada a dos animais que receberam dietas AC, porém, não houve efeito do nível de concentrado nos demais tempos de maturação (Tabela 2). Em relação a esses resultados, poderia ser esperado que os animais alimentados com dietas de alto concentrado apresentassem uma carne mais macia, o que normalmente ocorre em função de um melhor acabamento de carcaça, evitando problemas de maciez devido ao encurtamento das fibras musculares pelo frio durante o resfriamento das carcaças. Entretanto, neste trabalho, os animais foram abatidos com um grau de acabamento semelhante $(\sim 5 \mathrm{~mm})$, reduzindo assim o efeito da variação da gordura subcutânea sobre a maciez da carne e, além disso, uma espessura de gordura de $5 \mathrm{~mm}$ seria suficiente para proteção das carcaças durante o resfriamento. Dessa forma, o fato de os animais do tratamento $\mathrm{BC}$ apresentarem menor $\mathrm{FC}$ não tem nenhuma explicação aparente.

Os SCAG não influenciaram a FC em nenhum dos tempos avaliados. Para todos os tratamentos, a FC diminui linearmente $(\mathrm{P}<0,0001)$ com o aumento do tempo de maturação. Esses resultados foram semelhantes aos relatados por AFERRI et al. (2005) e SILVAet al. (2007), que também não observaram efeito da adição de SCAG sobre a maciez da carne de novilhos terminados em confinamento.

\section{CONCLUSÃO}

Dietas com elevada proporção de concentrado e a adição de sais de cálcio de ácidos graxos melhoraram as características quantitativas (peso e rendimento) das carcaças de bovinos cruzados em fase de terminação. Esse resultado faz com que essas carcaças possam ser usadas pelos produtores como alternativa para melhorar a eficiência do sistema de produção, uma vez que essas características são utilizadas pela indústria para a remuneração dos produtores. No entanto, é importante avaliar se o custo decorrente da utilização dessas ferramentas não sobrepõe os ganhos em desempenho.

\section{AGRADECIMENTOS}

Os autores agradecem à Cia Açucareira Vale do Rosário (Santeliza Vale Bioenergética S/A), pelo suporte necessário à condução deste trabalho.

\section{COMITÊ DE ÉTICA E BIOSSEGURANÇA}

Todos os procedimentos envolvendo os animais utilizados neste estudo foram realizados de acordo com as normas estabelecidas pelo Comitê de Ética em Experimentação Animal da Faculdade de Medicina Veterinária e Zootecnia da Universidade de São Paulo (Proc. 2098/2010).

\section{REFERÊNCIAS}

AFERRI, G. et al. Desempenho e características de carcaça de novilhos alimentados com dietas contendo diferentes fontes de lipídios. Revista Brasileira de Zootecnia, v.34, n.5, p.1651-1658, 2005. Disponível em: <http://www.scielo.br/scielo.php?script=sci_arttext\&pid=S151635982005000500027\&lng=pt\&nrm=iso>. Acesso em: 29 nov. 2010. doi: 10.1590/S1516-35982005000500027.

ALMEIDA, R. et al. Fazendas de terminação. In: PIRES, A.V. Bovinocultura de corte. Piracicaba: FEALQ, 2010. Cap.11, p.183-199. 
AMERICAN MEAT SCIENCE ASOCIATION (AMSA). Research guidelines for cookery, sensory evaluation, and instrumental tenderness of fresh meat. Chicago, IL: American Meat Science Association and National Live Stock and Meat Board, 1995. 49p.

BULLE, M.L.M. et al. Desempenho de tourinhos cruzados em dietas de alto teor de concentrado com bagaço de cana-de-açúcar como único volumoso. Revista Brasileira de Zootecnia, v.31, n.1, p.444-450, 2002 (supl.). Disponível em: <http://www.scielo.br/ scielo.php? script $=$ sci_arttext \& pid=S 1516 $35982002000200020 \& \operatorname{lng}=$ pt\&nrm=iso>. Acesso em: 29 nov. 2010. doi: $10.1590 /$ S1516-35982002000200020.

CROWDER, M.J.; HAND, D.J. Analysis of repeated mesures. London: Chapman \& Hall, 1990. 257p.

LANNA, D.P.D.; PACKER, I.U. Eficiência biológica e econômica de bovinos de corte. In: WORKSHOP SOBRE QUALIDADE DA CARNE E MELHORAMENTO GENÉTICO DE BOVINOS, 1998, São Carlos, SP. Anais... São Carlos: Embrapa-CPPSE/São Paulo: FUNDEPEC/Campo Grande: Embrapa-CNPGC, 1998. p.172.

LEME, P.R. et al. Utilização do bagaço de cana-de-açúcar em dietas com elevada proporção de concentrados para novilhos Nelore em confinamento. Revista Brasileira de Zootecnia, v.32, v.6, p.1786-1791, 2003. (Supl.1). Disponível em: <http:/ /www.scielo.br/scielo.php?script=sci_arttext\&pid=S1516$35982003000700029 \& \operatorname{lng}=$ pt\&nrm=iso>. Acesso em: 29 nov. 2010. doi: 10.1590/S1516-35982003000700029.

MILLEN, D.D. et al. A snapshot of management practices and nutritional recommendations used by feedlot nutritionists in Brazil. Journal of Animal Science, v.87, p.3427-3439, 2009.

NGIDI, M.E. et al. Effects of calcium soaps of long-chain fatty acids on feedlot performance, carcass characteristics and ruminal metabolism of steers. Journal of Animal Science, v.68, p.2555-2565, 1990. Disponível em: <http://jas.fass.org/cgi/content/full/87/10/ 3427 ? maxtoshow $=$ \&hits $=10 \&$ RESULTFORMAT $=\&$ author $1=$ mill en\&searchid $=1 \&$ FIRSTINDEX $=0$ \& resourcetype $=$ HWCIT $>$. Acesso em: 29 nov. 2010. doi:10.2527/jas.2009-1880.

OWENS, F.N.; GARDNER, B.A. A review of the impact of feedlot management and nutrition on carcass measurements of feedlot cattle. Journal of Animal Science, v.77, p.1-18, 2000. Disponível em: <http://jas.fass.org/cgi/reprint/77/E-Suppl/ $1 \mathrm{ag}$ ? maxtoshow $=$ \&hits $=10$ \&RESULTFORMAT $=$ \&author $1=$ owen $\mathrm{s}+\&$ fulltext=review\&andorexactfulltext $=$ and $\&$ searchid $=1 \&$ FIRSTIN DEX $=0 \&$ sortspec $=$ relevance $\&$ resourcetype $=$ HWCIT $>$. Acesso em: 29 nov. 2010.

PUTRINO, S.M. et al. Desempenho de tourinhos Brangus e Nelore alimentados com diferentes proporções de concentrado. In: REUNIÃO ANUAL DA SOCIEDADE BRASILEIRA DE ZOOTECNIA, 34., 2002, Recife, PE. Anais... Recife: Universidade Federal de Pernambuco, 2002. 1 CD.

SILVA, S.L. et al. Milho grão seco ou úmido com sais de cálcio de ácidos graxos para novilhos Nelore em confinamento. Revista Brasileira de Zootecnia, v.36, n.5, p.1426-1434, 2007. Disponível em: <http://www.scielo.br/scielo.php?script=sci_arttext\&pid=S151635982007000600028\&lng=pt\&nrm=iso>. Acesso em: 29 nov. 2010. doi: $10.1590 / \mathrm{S} 1516-35982007000600028$.

VALINOTE, A.C. et al. Fontes de lipídeos e monensina na alimentação de novilhos nelore e sua relação com a população de protozoários ciliados do rúmen. Revista Brasileira de Zootecnia, v.34, n.4, p.1418-1423, 2005. Disponível em: <http:/ $/ \mathrm{www}$. scielo.br/scielo.php? script=sci_arttext $\&$ pid $=\mathrm{S} 1516$ $35982005000400040 \& \operatorname{lng}=$ pt\&nrm=iso $>$. Acesso em: 29 nov. 2010. doi: 10.1590/S1516-35982005000400040.

ZINN, R.A. et al. Influence of ruminal biohydrogenation on the feeding value of fat in finishing diets for feedlot cattle. Journal of Animal Science, v.78, p.1738-1746, 2000. Disponível em: <http://jas.fass.org/cgi/reprint/78/7/ 1738? maxtoshow $=$ \&hits $=10 \&$ RESULTFORMAT $=$ \&author $1=$ zinn \& andorexactfulltext $=$ and \&searchid $=1 \&$ FIRSTINDEX $=0 \&$ sortspec $=$ relevance $\&$ resourcetype $=$ HWCIT. Acesso em: 29 nov. 2010 .

WEISS, W.P. et al. A theoretical-based model for predicting total digestible nutrient values of forages and concentrates. Animal Feed Science and Technology, v.39, p.95-110, 1992. Disponível em: <http://www.sciencedirect.com/ science?_ob=MImg\&_imagekey=B6T 42-49N8SX4-1C$1 \&$ \&cdi $=4962 \&$ _user $=5674931 \&$ _pii $=0377840192900344 \&$ \&o

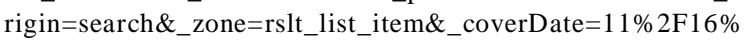
2 F 1992 \&_sk $=999609998 \& \mathrm{wch} \mathrm{p}=\mathrm{d} \mathrm{G} \mathrm{L} \mathrm{z} \mathrm{V} \mathrm{lb} \mathrm{-}$ zSkzk\&md5=6694e24267c94774e927d794e05d6a4b\&ie=/ sdarticle.pdf. 